\title{
LAS ESTRUCTURAS Y EL PESO PROPIO
}

\section{(THE STRUCTURES AND THE DEADLOAD)}

Jaime Cervera Bravo, Dr. Arquitecto

Instituto Juan de Herrera. ETS de Arquitectura

MADRID/ESPAÑA
400-22

Fecha de recepción: 17-1-90

\section{RESUMEN}

En las estructuras pueden distinguirse con bastante precisión los aspectos de su comportamiento que dependen sólo de la forma, de su proporción, de aquellos que dependen de su tamaño. Mediante el análisis de estructuras en el límite de resistencia a su propio peso, y a través de los conceptos de esbeltez, alcance del material, alcance de la estructura y de cantidad de estructura de la "estructura unidad" pueden delimitarse con mucha precisión dichos aspectos. De este modo en estructuras muy lejos del límite de tamaño correspondiente a su tipo, las relaciones de proporción son dominantes en su comportamiento. En estructuras con tamaño importante respecto del límite, los conceptos anteriores proporcionan recursos para evaluar con sencillez y elegancia las proporciones de la resistencia del material que se emplean en soportar el propio peso, o las cargas adicionales a éste.

\section{SUMMARY}

We can distinguish rather precisely between the aspects of structural comportment dues to it's form, to it's proportions, from those dues to it's size. By the analysis of structures in their size's limits (supporting only their own's weight), and with the use of the slenderness, scope of the material, scope of the structure, and structure quantity of the "unit structure" concepts, we can delimit those aspects. Thus, in structures far from the size's limit that can be adscribed to it's class, the relations of the form are dominants in their comportement. In structures where the size is a high fraction of that limit, these concepts allow to evaluate with quickness and elegance the fractions of strehgth employed in supporting the structure's own weight, or any additional loads.

\section{INTRODUCCIÓN}

En la antigüedad clásica se concebía el comportamiento de las estructuras como un fenómeno principalmente ligado a la forma, a la geometría, que era la ciencia matemática con mayor desarrollo. Tal concepción sugería el uso de reglas proporcionales en el diseño, de modo que modelos probados en un tamaño se extrapolaban mediante reglas de proporción a otros tamaños. Galileo demostró usando geometría que las reglas proporcionales no eran apropiadas a estructuras soportando su propio peso, y su alegato tuvo una influencia importante. Las razones de Galileo, y el desarrollo y uso del análisis matemático como herramienta de reflexión estructural han eliminado casi por completo las reglas de proporción de la teoría de las estructuras. Hay que salvar los casos puntuales en que las expresiones se plantean en forma adimensional, que podría verse como una forma actual de establecer reglas proporcionales desprovistas de la carga geométrica (visual) de la antigüedad. Hay que decir que, de encontrarse reglas proporcionales válidas, éstas son una poderosa herramienta de diseño, en la medida en que éste se resuelve mediante dibujos en los que se trata de dar so- luciones a todos los problemas implicados. La herramienta tiene así la misma forma del medio de trabajo empleado en el propio proceso del diseño, y es de gran utilidad.

Si analizamos "grosso modo" el comportamiento de estructuras en función del tipo de carga que soportan, es fácil intuir que estructuras diseñadas para soportar cargas que dependan de superficies llevarán a soluciones proporcionales, en la medida en que aumentos paralelos de las áreas de carga y de sección, para esquemas estructurales idénticos y formas y secciones proporcionales mantendrán las tensiones en los materiales. Esta es la base de cantidad de diseños (proporcionales) de uniones o detalles. No aportaré mayor precisión aquí, pues no es éste mi objetivo. Para cargas dependientes de masas, en cambio, y en particular para los pesos propios de las estructuras, la proporcionalidad no es aplicable, de modo que la geometría parece inútil como herramienta. En este artículo mostraré que aun en este caso puede ser una herramienta de excepcional valor. 
En los manuales académicos no es usual encontrar un análisis teórico del comportamiento de las estructuras sometidas a su propio peso (ni por tanto de su diseño), que no sea el hecho de citar su importancia (mayor o menor) en la evaluación de las cargas. De este modo no es fácil hacerse una idea general de la incidencia del peso en función del tipo de estructura, su forma, su tamaño, o el material de que esté compuesta, hasta que ésta no está diseñada. Tal idea queda limitada a la que pueda aportar la experiencia del proyectista en estructuras anteriores. Como la experiencia no es susceptible de una fácil transmisión, ni de generalización, el conocimiento asociado a ésta mantiene un carácter de relativa oscuridad, que no es una cualidad deseable en él. Es objetivo de este artículo iluminar algo dicha oscuridad mediante una reflexión sobre la incidencia del peso propio en el comportamiento y diseño de las estructuras. Muchos de los resultados que aqui se exponen proceden de reflexiones elaboradas a partir del curso de doctorado impartido por Ricardo Aroca: "Geometría y Proporción en el Diseño de Estructuras".

Describimos en primer lugar, para centrar ideas, las condiciones de proporcionalidad en el comportamiento de las vigas. Analizamos posteriormente el comportamiento de las estructuras en el límite teórico de su resistencia (sólo soportan su propio peso), estableciendo de paso un lenguaje apropiado para referirnos al mismo, tratando finalmente el de estructuras con reserva de resistencia para cargas adicionales a aquél.

\section{Proporcionalidad en el comportamiento de vigas}

Describimos las condiciones geométricas de resistencia y de deformación limitada en vigas de sección rectangular constante para carga uniforme y peso propio.

En cortante, momento y deformación, las expresiones de comprobación son del tipo:

$$
\begin{array}{ll}
T=\alpha_{1}(q s+b d \varrho) / / 2 & \leq \beta_{1} \tau b d \\
M=\left.\alpha_{1}(q s+b d \varrho)\right|^{2} / 8 & \leq \beta_{2} b d^{2} \sigma \\
f / I=\left.\alpha_{3}(q s+b d \varrho)\right|^{4} /(384 E I I) & \leq \beta_{3}
\end{array}
$$

Con:

- q: Carga por metro cuadrado.

- I $\neq$ I: Luz de la viga.

- s: Separación entre vigas (o luz de carga).

- d: Canto de la viga

- b: Ancho de la viga.

- $\mathrm{:} \mathrm{Densidad} \mathrm{del} \mathrm{material.}$

- $\sigma$ : Resistencia a tensión normal del material.

- $\tau$ : Resistencia a tensión tangencial del material.

- E: Módulo de elasticidad del material.

- I: Inercia de la sección (bd $3 / 12)$.

- $\alpha_{1}$ : Factor por condición de extremo en el cortante (1 en condiciones simétricas).

- $\alpha_{2}$ : Factor por condición de extremo en el momento ( 1 en vigas apoyadas, 0,666 en empotradas elásticas, 0,5 en empotradas plásticas,...).

- $\alpha_{3}$ : Factor por condición de extremo en deformación (1 en vigas empotradas, 5 en vigas apoyadas...).

- $\beta_{1}$ : Factor de resistencia de la sección en cortante $(0,66$ para vigas de comportamiento elástico, 0,9 para secciones de hormigón,...).

- $\beta_{2}$ : Factor de resistencia de la sección en flexión (0,1666 para vigas de comportamiento elástico, 0,255 para secciones de hormigón con cuantía mecánica $0,3,0,353$ si la cuantía es de $0,45, \ldots$.$) .$

- $\beta_{3}$ : Límite de flecha admitido $(1 / 400, \ldots)$.

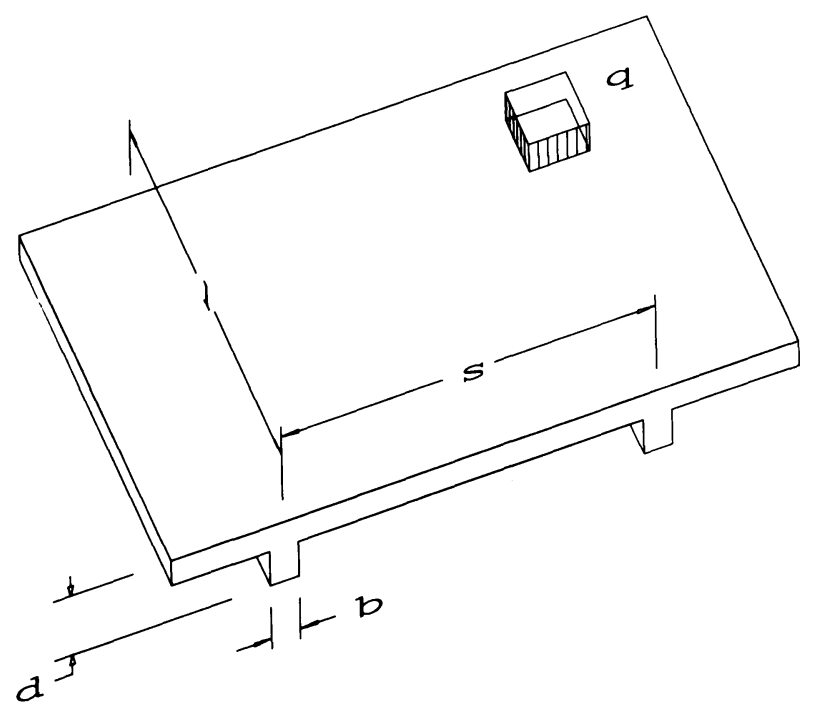

Fig. 1.-Módulo de diseño.

Si ordenamos adecuadamente las expresiones anteriores, resulta:

$$
\begin{aligned}
& \frac{\mathrm{l} s}{\mathrm{~d} b} \leq 2 \frac{\beta_{1}}{\alpha_{1}} \frac{\tau}{q} \frac{1}{1+\frac{d \varrho}{q s / b}} \\
& \frac{\beta^{2} s}{d^{2} b} \leq 8 \frac{\beta_{2}}{\alpha_{2}} \frac{\sigma}{q} \frac{1}{1+\frac{d \varrho}{q s / b}} \\
& \frac{\beta^{3} s}{d^{3} b} \leq 384 \frac{\beta_{3}}{\alpha_{3}} \frac{E}{q} \frac{1}{1+\frac{d \varrho}{q s / b}}
\end{aligned}
$$


Llamamos $\Gamma_{\mathrm{s}}$ al cociente $\mathrm{s} / \mathrm{b}$ y $\Gamma_{\mathrm{d}}$ al cociente $\mathrm{l} / \mathrm{d}$.

$\Gamma_{\mathrm{d}} \Gamma_{\mathrm{s}} \leq \mathrm{K}_{1} \pi \mathrm{q} \mathrm{F}$

$\Gamma_{\mathrm{d}}^{2} \Gamma_{\mathrm{s}} \leq \mathrm{K}_{2} \sigma / \mathrm{q} \mathrm{F}$

$\Gamma_{\mathrm{d}}{ }^{3} \Gamma_{\mathrm{s}} \leq \mathrm{K}_{3}$ E/q F

Expresiones adimensionales en las que los cocientes del primer miembro son esbelteces (proporciones), y los términos que intervienen en el segundo son en gran medida dependientes sólo del tipo de problema: En efecto, en $\mathrm{K}_{i}$ se consideran sólo terminos del problema pero no de las dimensiones de la solución. En $F$ se incluyen sólo los términos dependientes de la relación entre carga y peso propio. Entre las tres comprobaciones existen unos valores límite (que separan los puntos de corte entre diseño por cortante, diseño por momento, o diseño por deformación, en la medida en que la solución no es estricta simultáneamente para las tres condiciones), límites que se obtienen por cociente entre las ecuaciones dos a dos (o haciendo estricta la solución a dos requerimientos simultáneamente), y que no dependen de las relaciones entre carga externa y peso propio que puedan existir.

$\Gamma_{\mathrm{b}}=\mathrm{K}_{2} / \mathrm{K}_{1} \sigma / \tau$; "Esbeltez base"(1)

$\Gamma_{1}=K_{3} / K_{2} E / \sigma$; "Esbeltez límite"(2)

Para extraer la influencia de cargas o pesos propios, anulamos alternativamente la densidad o la carga externa, obteniendo las condiciones geométricas de d:seño para vigas sin peso propio, o vigas sin carga en el límite de tamaño.

En el primer caso $F=1$, y en el segundo $F / q=$ $=\Gamma_{\mathrm{s}}$ ld $\varrho$ de modo que, para estructuras sin peso propio, las comprobaciones son:

$\Gamma_{\mathrm{d}} \Gamma_{\mathrm{s}} \leq \mathrm{K}_{1} \pi \mathrm{q}=\mathrm{K}_{\mathrm{T}}$

$\Gamma_{\mathrm{d}}^{2} \Gamma_{\mathrm{s}} \leq \mathrm{K}_{2} \sigma / \mathrm{q}=\mathrm{K}_{\sigma}$

$\Gamma_{\mathrm{d}}^{3} \Gamma_{\mathrm{s}} \leq \mathrm{K}_{3} \mathrm{E} / \mathrm{q}=\mathrm{K}_{e}^{(3)}$

Como se ve se trata de estructuras en las que existe proporcionalidad entre las soluciones, pues las condi- ciones de comprobación (que son el valor de las esbelteces, y por tanto proporciones geométricas) sólo dependen de condiciones ajenas al propio dimensionado.

Para estructuras sin carga externa las expresiones pasan a ser las siguientes:

$$
\begin{aligned}
& \mathrm{I} \leq \mathrm{K}_{1} \pi \sigma \sigma / \varrho \\
& \mid \Gamma_{\mathrm{d}}^{2} \leq \mathrm{K}_{2} \sigma / \varrho \\
& I \Gamma_{d} \leq K_{3} E / \sigma \sigma / \varrho
\end{aligned}
$$

Donde l es la luz de la solución. Tales expresiones permiten, por lo tanto, evaluar la máxima luz alcanzable. Para dimensiones menores a la máxima, la parte de tensión utilizada para resistir el propio peso es igual a la proporción entre la luz real empleada y el tamaño máximo. Por lo tanto, si consideramos que $\sigma / \varrho$ es una longitud que depende del material elegido, y que los demás términos en $\mathrm{K}_{1}$ dependen sólo del problema y tipo de solución elegidos, se comprenderá el interés del tipo de análisis empleado: permite abordar las comprobaciones en forma geométrica, empleando términos de forma, esbeltez, tamaño...

Puede alegarse que no es posible describir desde la geometría el comportamiento de las estructuras que sólo resisten su propio peso y que han sido diseñadas estrictamente para tal función, al contrario del caso abordado hasta aquí, de estructuras que por ser de sección constante son ineficientes para tal función, y que, por tanto, no tienen por qué representar el comportamiento de las estructuras correspondientes a lo que sería una clase diferente de problemas. Sin embargo sí es posible tal descripción, y a ello dedicamos el siguiente apartado.

(1)Término utilizado desde hace años por José Luis de Miguel para denominar la esbeltez que separa los campos de diseño por cortante y por momento.

(2)Separa los campos de diseño por momento y por deformación. Incidentalmente cabe añadir que para ciertos tipos (extraños) de diseño sobredimensionados a flexión podría darse el caso de una esbeltez límite entre diseño por cortante y diseño por deformación, que se obtendria de análoga forma a las anteriores.

${ }^{(3)}$ Términos debidos a Ricardo Aroca, en sus cursos de Proyectos de Estructuras en la Escuela de Arquitectura de Madrid.

\section{Estructuras que resisten su propio peso}

En el presente apartado se describirá el comportamiento de las estructuras sometidas a su propio peso, y en el límite de su resistencia, analizando el diseño de su forma, y evaluando el tamaño que pueden alcanzar, intentando determinar los parámetros claves en tales cuestiones.

En todos los casos, para tales tipos de estructuras (y en recuadro aparte se consideran el soporte, el arco, o las vigas de canto variable, sin alma, o de canto cons(c) Consejo Superior de Investigaciones Científicas Licencia Creative Commons 3.0 España (by-nc) tante, pero puede observarse análogamente en los casos considerados al analizar la proporcionalidad en el comportamiento de vigas), aparecen dos resultados de capital importancia:

En primer lugar, las expresiones dependen directamente de la longitud $\sigma / \varrho$ (en el caso del soporte véase la altura para la que se duplica el área, en vigas, la propia expresión del tamaño máximo....). Puede decirse que so. luciones en las que sólo varíe el material son proporhttp://informesdelaconstruccion.revistas.csic.es 
cionales a los correspondientes valores de los materiales empleados. Denominamos Alcance ${ }^{4}$ a tal cantidad, en la medida en que tal valor es un tamaño, ligado intimamente a las máximas dimensiones alcanzables mediante el empleo de un cierto material, y que corresponde a la máxima longitud que puede alcanzar una barra de sección constante de un material sometida axilmente a su propio peso (y si no existen otros problemas, como serían la inestabilidad en compresión, o la necesidad de ejecución de uniones en tracción). En algunos textos se le ha denominado "altura máxima". Preferimos una denominación más sintética (más corta, y que actualmente tiene menor contenido semántico), que permita su incorporación natural al lenguaje con el que se evalúan y describen los contenidos estructurales de materiales, diseños concretos, etcétera.

En segundo lugar, en todos los casos las dimensiones de las secciones empleadas mantienen una relación definida entre sí, pero ellas mismas no están definidas, es decir, las formas y alcances logrados con ellas son independientes del dimensionado, por lo que pueden alterarse proporcionalmente todas las dimensiones sin que se produzca alteración ninguna en las restantes características del diseño. De modo que, con respecto al propio peso exclusivamente, aumentar las dimensiones de las secciones sin modificar la forma general no supone alteración en el estado de tensiones de cada sección: el aumento de sección es paralelo al incremento en el propio peso, por lo que no hay cambio alguno: las expresiones obtenidas son independientes de la dimensión de la sección utilizada como referencia en el análisis. De este modo, tal dimensión de referencia, desde el punto de vista del propio peso es irrelevante, si bien para la consideración de excesos de carga respecto a tal peso es fundamental. A la variación en el dimensionado de dicha sección de referencia la denominamos "grosor" o "espesor"5 de la estructura, de modo que las estructuras pueden ser "espesas" ("gruesas") o "finas" según que las dimensiones generales de sus secciones sean mayores o menores.

Si bien para el soporte no existe un límite teórico de tamaño (y de hecho el Everest es una prueba fehaciente de tal aserto), sí existe tal límite para las estructuras de mayor interés: las que resuelven problemas de flexión (o de traslado transversal de cargas).

En éstas el tamaño máximo puede evaluarse en función del Alcance del material empleado, de la Esbeltez de la forma, y de un parámetro dependiente de la propia forma en sí, del tipo estructural empleado, del esquema de estructura seleccionado para resolver el problema, factor que denomino factor de alcance del esquema, del tipo estructural empleado. Así, de las expresiones vistas más arriba puede obtenerse, para cada una de las comprobaciones citadas, el límite de tamaño

(c) Consejo Superior de Investigaciones Científicas Licencia Creative Commons 3.0 España (by-nc)

$$
\begin{array}{llr} 
& \leq \mathrm{K}_{1} & \pi \sigma \sigma / \varrho \\
\mathrm{I} & \leq \mathrm{K}_{2} / \Gamma_{\mathrm{d}} & \sigma / \varrho \\
\mathrm{I} & \leq \mathrm{K}_{3} / \Gamma_{\mathrm{d}}{ }^{2} & \text { El } \sigma \sigma / \varrho
\end{array}
$$

Si seleccionamos la expresión de la comprobación en flexión, resulta:

$$
\mathrm{I} \leq \Omega / \Gamma \sigma / \varrho
$$

con $\Omega$ con los valores siguientes para sección biapoyada, según diseño (todos con sección rectangular constante):

1,33: Diseño elástico para material isótropo.

2,04: Sección de hormigón con cuantía mecánica 0,3. 2,82: Sección de hormigón con cuantía mecánica 0,45 .

Asimismo, para las Vigas de Canto variable (sin alma) o de canto constante puede obtenerse la expresión (ver Anexo para su análisis en detalle):

$$
\mathrm{I} \leq \Omega / \Gamma \sigma / \varrho
$$

ver la expresión [10] del Anexo para canto variable, idéntica a la que puede establecerse para canto constante, donde sólo varían (en escasa medida) las expresiones de $\Omega$ en función de $\Gamma$, según las tablas siguientes, en las que:

$\Gamma$ es la esbeltez,

C es la luz máxima (correspondiente al signo igual en las inecuaciones anteriores); es el Alcance,

$\Omega$ es el llamado factor del alcance del esquema estructural, función del esquema, y relativamente estable con $\Gamma$,

$W$ es es el cociente $\mathrm{C} / \Gamma$,

$\mathrm{R}$ es el alcance del material $\sigma / \varrho$.

Tabla A

\begin{tabular}{|l|r|r|r|r|r|r|l|}
\hline \multicolumn{10}{|c|}{ ALCANCES de VIGA ESTRICTA de } \\
CANTO VARIABLE \\
\hline$\Gamma$ & 1 & 2 & 5 & 10 & 20 & 50 & Esbeltez \\
\hline$W$ & 2,58 & 1,73 & 0,779 & 0,397 & 0,200 & 0,080 & $\begin{array}{l}\text { Factor de } \\
\text { alcance del } \\
\text { esquema }\end{array}$ \\
\hline$\Omega$ & 2,58 & 3,46 & 3,89 & 3,97 & 4,00 & 4,00 & \\
\hline$C$ & 2,580 & 1,730 & 779 & 397 & 200 & 80 & $\begin{array}{l}\text { Alcance para } \\
\text { R }=1.000 \mathrm{~m}\end{array}$ \\
\hline
\end{tabular}

Tabla A

\begin{tabular}{|r|r|r|r|r|r|r|l|}
\hline \multicolumn{10}{|c|}{ ALCANCES de VIGA ESTRICTA de } \\
CANTO CONSTANTE \\
\hline$\Gamma$ & 1 & 2 & 5 & 10 & 20 & 50 & Esbeltez \\
\hline$W$ & 2,00 & 1,36 & 0,73 & 0,42 & 0,23 & 0,095 & $\begin{array}{l}\text { Factor de } \\
\text { alcance del }\end{array}$ \\
\hline$\Omega$ & 2,00 & 2,72 & 3,63 & 4,15 & 4,50 & 4,75 & \begin{tabular}{l} 
esquema \\
\hline$C$
\end{tabular} \\
2,000 & 1,360 & 730 & 420 & 230 & 95 & $\begin{array}{l}\text { Alcance para } \\
\mathrm{R}=1.000 \mathrm{~m}\end{array}$ \\
\hline
\end{tabular}

http://informesdelaconstruccion.revistas.csic.es 
Cabe señalar incidentalmente que entre las soluciones de viga evaluadas, la solución de viga de canto constante es más eficiente que la de sólo cordones con canto variable a partir de una esbeltez igual a 7,4273 (supuesto el uso del mismo material en ambas).

$$
\Gamma_{\mathrm{t}}=7,4273
$$

siendo los cordones paralelos más apropiados para esbelteces mayores que $\Gamma_{t}$.

Podemos trazar finalmente como comparación una gráfica del factor $\Omega=I \Gamma / R=I \Gamma \rho / \sigma$ para los casos de vigas estrictas y el de un posible tipo de viga de hormigón con cuantía mecánica 0,30 en sección rectangular constante.

Vemos, pues, que pueden obtenerse análogas conclusiones geométricas sobre los tamaños máximos (alcances) para estructuras diseñadas a peso propio, conclusiones válidas tanto si se diseña con criterios de sección constante como si se hace con criterios de sección estricta.

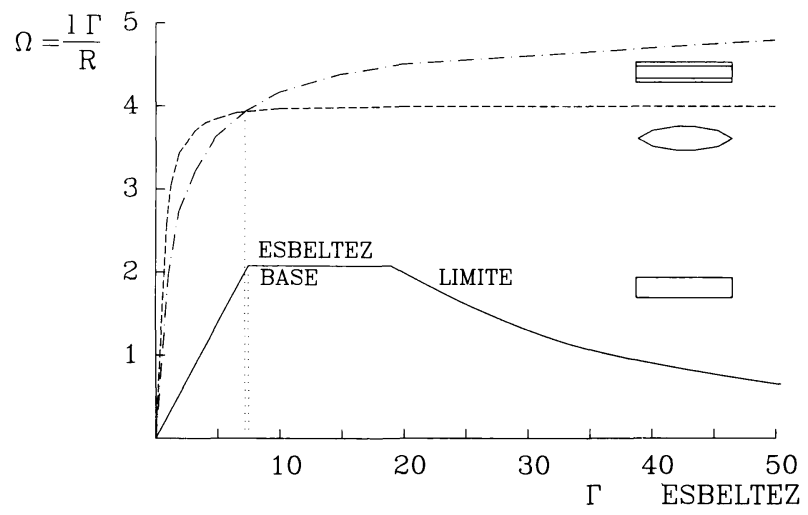

Fig. 2.-Límites de tamaño según esbeltez.

(4) A inicios del curso 1989.90 propuse el uso de dicha denominación para su empleo en el curso de Proyectos de Estructuras de la ETSAM. Parece probada (experimentalmente) la idoneidad de dicha denominación.

${ }^{(5)}$ Se trata de un muy importante concepto, destacado y bautizado por Ricardo Aroca en el curso de Doctorado citado con anterioridad.

\section{ESTRUCTURAS CON RESERVA DE RESISTENCIA ADICIONAL}

Una estructura de tamaño menor al alcance de la solución utilizada, es decir, de tamaño menor al máximo posible para el diseño empleado (alterado en forma proporcional), está sometida, bajo el propio peso, a unas tensiones que están en proporción con respecto a las de la estructura máxima en la misma relación que el tamaño real de la estructura es al alcance de ésta. Pues en efecto, al reducir el tamaño, las cargas de peso propio reducen con el cubo de la disminución de dimensiones, mientras que las secciones reducen con el cuadrado de éstas. De este modo puede evaluarse con extremada facilidad la reserva de resistencia que puede utilizarse para soportar carga externa.

Si llamamos $C$ al alcance de la solución empleada y C al tamaño de la estructura, las tensiones por peso propio valdrán $\mathrm{C} / \mathrm{C}$ de las de la estructura máxima, y si ésta era estricta para peso propio, valdrán $\sigma \mathrm{C} / \mathrm{C}$. (Si no era de diseño estricto para peso propio se tendrá ese valor en el punto peor, siendo menores las tensiones en el resto de la estructura). La reserva de resistencia es de:

$$
\sigma(1-c / C)
$$

Vamos a considerar ahora un enfoque diferente utilizando el concepto de "Cantidad de estructura"6. Una estructura sometida a carga externa tiene un consu- mo estructural que puede medirse por la "Cantidad de estructura",

$$
W=\int|N| d s=\alpha Q c
$$

siendo $\alpha$ un cierto factor de forma, $Q$ la carga total (su. ma de todas ellas) y $c$ el tamaño de la estructura.

Considérese despacio el papel del factor de forma $\alpha$ : su valor es el de la cantidad de estructura consumida en una solución estructural proporcional a la del problema propuesto, para tamaño unidad y carga total unidad. Corresponde, pues, a un coeficiente de significado preciso, e interesante: la cantidad de estructura de la "estructura unidad" correspondiente a la solución elegida.

Si la estructura es estricta, de un cierto material, el peso de la estructura vale su volumen por su densidad, es decir,

$$
\mathrm{P}=\varrho \mathrm{V}=\varrho \mathrm{W} / \sigma=\mathrm{W} \varrho / \sigma=\alpha \mathrm{Qc} \varrho / \sigma
$$

Para estructuras no estrictas podemos considerar el exceso de material sobre la estricta incorporado en el término $\alpha$ (como un factor de sobre-consumo respecto de la estricta) de modo que la expresión siga sien- 
do válida en todo tipo de estructura sometido a carga externa. Si el peso propio puede considerarse en todas partes como parte alícuota (constante) de la carga externa (es decir, si la ley de carga es proporcional a la ley de cargas que define el peso propio) resultará que la carga total vale:

$Q=Q_{0}+\alpha Q c \rho / \sigma$

$\mathrm{Q}(1-\alpha \mathrm{C} \varrho / \sigma)=\mathrm{Q}_{0}$

$Q=\frac{Q_{0} 1}{(1-\alpha C \varrho / \sigma)}$

y el factor resultante puede considerarse como un factor de ampliación de carga que permite obtener el valor de la carga total a partir del de la carga, sin incluir el peso propio de la estructura. Dicho factor de ampliación de carga es, pues,

$$
\frac{1}{1-d c \rho / \sigma}
$$

El inverso de dicho factor puede verse también como un factor de reducción de la tensión admisible de cara a considerar el peso propio, es decir, es el factor correspondiente a la reserva de resistencia de la estructura, de modo que comparando con las expresiones del apartado anterior resultará:

$$
1-\alpha \mathrm{c} \varrho / \sigma=1-\mathrm{c} / \mathrm{C}
$$

de donde también puede obtenerse

$$
\begin{aligned}
& \mathrm{C} / \mathrm{C}=\alpha \mathrm{c} \rho / \sigma \\
& \mathrm{C}=\alpha \varrho 1 / \alpha
\end{aligned}
$$

y recordando la expresión [10] del Anexo

$C_{\varrho} / \sigma=1 / \alpha=\Omega / \Gamma$

Puede llegarse a idéntica expresión sin más que pensar que para un tamaño igual al alcance de la estructura el factor de ampliación de carga ha de ser infinito: una carga inicial nula supone una carga total (igual al peso de la estructura) finita, de modo que el denominador de [2] debe anularse en ese caso.

De esta última expresión pueden extraerse, para este caso, las conclusiones siguientes:

a) El alcance del material dividido por el alcance de la estructura no es más que la cantidad de estructura de la "estructura unidad".

b) Dicha cantidad de estructura vale la esbeltez dividida por el factor de alcance de la solución.

c) Dicha cantidad define asimismo la relación entre carga total sobre la estructura y peso propio, en la forma de la expresión [1].

\section{CONCLUSION}

El peso propio de las estructuras puede ser considerado de forma consistente mediante el empleo de los conceptos de Alcance y Cantidad de Estructura, conceptos que para cada Solución Estructural y Material se encuentran íntimamente ligados.

En estructuras sin peso propio, con cargas asociadas a superficies, pueden en general formularse las expresiones de comprobación en términos geométricos, o de proporciones de forma.

En estructuras que se soportan a sí mismas, sin carga adicional alguna, pueden formularse las expresiones de comprobación en términos de límite de tamaño, dependientes de la forma y el material empleado.

Tal límite de tamaño, o alcance, puede medirse básicamente en función del alcance del material empleado (o longitud máxima de la barra de sección constante que se soporta a sí misma a esfuerzo axil simple), dividido por la esbeltez de la forma utilizada, y multiplicada por un factor de forma que depende del tipo empleado en la solución (y en menor medida de la esbeltez).
La parte de tensiones dedicadas a soportar el peso propio de las estructuras con reserva de resistencia a cargas adicionales a su propio peso es igual al cociente entre el tamaño de la estructura y-el alcance de la solución utilizada.

Dicha proporción, así como el alcance de las estructuras son independientes del dimensionado de la estructura, de su "espesor", si tal dimensionado mantiene en todas las secciones las relaciones correspondientes a la estructura en el límite de su tamaño.

Dicha proporción, en estructuras de ley de carga proporcional a la ley de peso propio, no es más que la cantidad de estructura correspondiente al caso estudiado para carga unidad dividido por el alcance del material empleado.

De este modo la propuesta que hacíamos al inicio de llevar a la geometría un peso importante del proceso de diseño puede cumplirse, habiéndose formalizado con sencillez el proceso de preevaluación del peso propio de las estructuras. 


\section{DISEÑO DE TIPOS ESTRUCTURALES SOMETIDOS SÓLO A PESO PROPIO}

Vamos a analizar en detalle varios casos concretos de estructuras diseñadas estrictamente para soportar su propio peso. Trataremos de tipologías sencillas, como son el soporte, el arco o la viga. Suponemos que los valores de resistencia del material, $\sigma^{(\lambda)}$, y densidad del material $\varrho$ están dados, y son constantes en toda la estructura.

(7) En este texto usamos sistemáticamente la noción de tensión admisible $\sigma$, por sencillez de notación.

\section{Soporte a peso propio}

Sea un corte de sección superior $A$, inferior $A+d A$, y altura dh. La Carga sobre la sección superior es $\sigma \mathrm{A}$

y sobre la sección inferior

$$
\sigma(A+d A)
$$

que ess igual a

$$
\sigma \mathrm{A}+\varrho \mathrm{Adh}
$$

de modo que

$$
\mathrm{dA}=\varrho / \sigma \mathrm{Adh}
$$

y por ende

$$
\mathrm{dA} / \mathrm{A}=\rho / \sigma \mathrm{dh}
$$

Intregrando la expresión resulta

$$
\ln \mathrm{A}=\mathrm{\rho} / \sigma \cdot \mathrm{h}
$$

es decir,

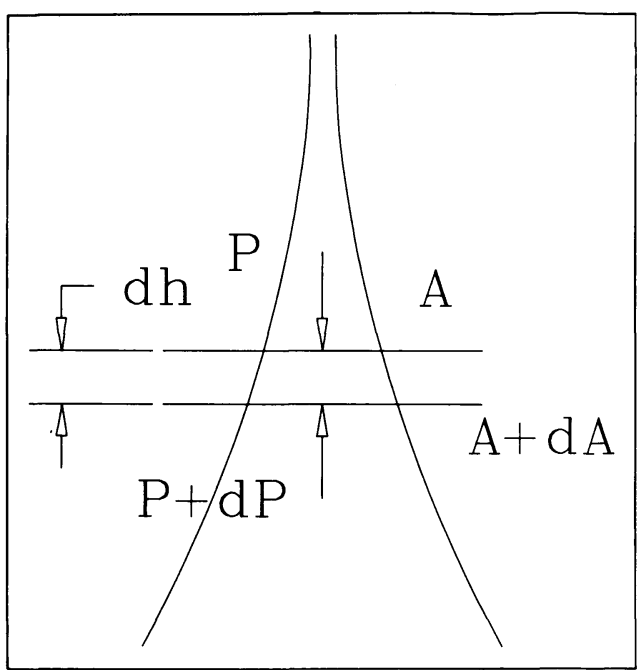

Fig. 3.-Soporte a peso propio.

$$
A=C e^{\left(e^{(\sigma) h} h\right.}
$$

Determinar el valor de la constante de integración no es difícil: basta tomar un origen de alturas $\mathrm{h}=0$, y la constante es el área en tal punto.

Por lo tanto, la expresión de la columna buscada es:

$$
A=A_{o} e^{(e / \sigma) h}
$$

El Area se duplica para

$$
\begin{aligned}
2 \mathrm{~A}_{0} & =\mathrm{A}_{0} \mathrm{e}^{(\mathrm{e} / \mathrm{\rho} \mathrm{h}} \\
\mathrm{h} & =\sigma \varrho \ln 2
\end{aligned}
$$

En el texto se analizan las implicaciones de estas expresiones.

\section{Arco sometido a su propio peso}

Sea un arco de radio de curvatura en la clave $R_{0}$. La curvatura mínima que puede lograrse viene dada por la ecuación de equilibrio:

$$
\begin{gathered}
\sigma \mathrm{A}_{0} \mathrm{~d} \theta=\varrho \mathrm{A}_{0} \mathrm{R}_{0} \mathrm{~d} \theta \\
\mathrm{R}_{0}=d \varrho
\end{gathered}
$$


Si consideramos una rebanada cualquiera tal como en la figura, para $\theta$ arbitraria, y evaluamos el equilibrio de fuerzas, tenemos, en componente vertical:

$\sigma \mathrm{Ad} \theta \cos \theta+\sigma \mathrm{dAsen} \theta=\varrho \mathrm{ARd} \theta$

$y$ en componente horizontal

$\sigma \mathrm{Ad} \theta \operatorname{sen} \theta=\sigma \mathrm{dA} \cos \theta$

que lleva a las dos ecuaciones siguientes para la rebanada:

$$
\begin{gathered}
d \theta \cos \theta+d A / A \operatorname{sen} \theta=\rho / \sigma R d \theta \\
\operatorname{tg} \theta d \theta=d A / A
\end{gathered}
$$

Integrando ésta,

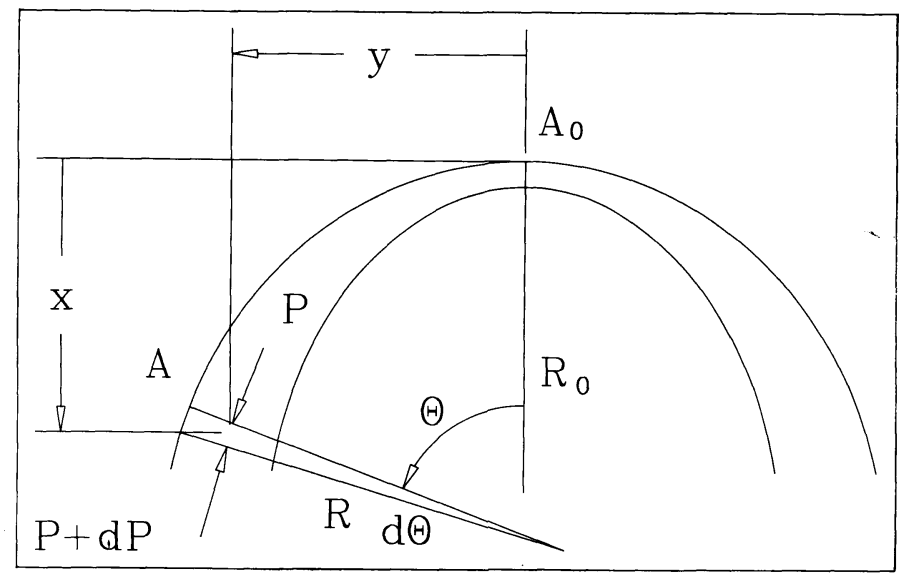

Fig. 4.-Arco a peso propio.

$\ln (C A)=-\ln (\cos \theta)=\ln (1 / \cos \theta)$

de donde

$$
A=A_{0} / \cos \theta ;
$$

y sustituyendo en la primera

$$
\mathrm{d} \theta / \operatorname{tg} \theta+\operatorname{tg} \theta \mathrm{d} \theta=\mathrm{R} / \mathrm{R}_{\mathrm{o}} \mathrm{d} \theta / \operatorname{sen} \theta
$$

(recordar [3] para el significado de $R_{0}$ ) de modo que

$$
R=R_{0}(\cos \theta+\operatorname{sen} \theta \operatorname{tg} \theta)
$$

es decir,

$$
\mathrm{R}=\mathrm{R}_{0} / \cos \theta
$$

Podemos ahora determinar la expresión de la curva:

$$
d x=R \cos \theta d \theta
$$

de modo que

$$
\mathrm{dx}=\mathrm{R}_{\mathrm{o}} \mathrm{d} \theta
$$

y por tanto

$$
\mathrm{x}=\mathrm{R}_{\mathrm{o}} \theta
$$

Además tenemos

$$
\mathrm{dy} / \mathrm{dx}=\operatorname{tg} \theta
$$

es decir

$$
d y=d x \operatorname{tg}\left(x / R_{0}\right)=-R_{0} d\left(\cos \left(x / R_{0}\right)\right) \div \cos \left(x / R_{0}\right)
$$

de modo que integrando

$$
y=R_{0} \ln \left(1 / \cos \left(x / R_{0}\right)\right)
$$

La apertura teórica máxima del arco corresponde a 2 veces la máxima $x$, de modo que

$$
C=2 X_{\max }=\pi R_{\circ}
$$

en este valor la y se hace infinita, por lo que se trata de un tamaño inalcanzable.

Al igual que con el soporte podemos evaluar el punto en el que el área se duplica sin más que ver

$$
\begin{aligned}
& A_{i}=A_{o} / \cos \theta_{i}=2 A_{j}=2 A_{0} / \cos \theta_{j} ; \cos \theta_{i}=\cos \theta_{j} / 2 ; \\
& R_{i}=2 R_{j}
\end{aligned}
$$




$$
\begin{aligned}
& y_{i}=R_{o} \ln \left(1 / \cos \theta_{i}\right) \\
& \left.y_{i}=R_{0} \ln / 1 / \cos \theta_{j}\right)=R_{0} \ln \left(1 / 2 \cos \theta_{i}\right) \\
& y_{i}=R_{o}\left(\ln \left(1 / \cos \theta_{i}\right)+\ln (1 / 2)\right)
\end{aligned}
$$

y la diferencia de altura entre secciones que se duplican es

$$
h=y_{j}-y_{i}=R_{0} \ln (1 / 2)
$$

De este modo hemos obtenido la forma general del arco sometido a su propio peso, en trazado $y(x)$ y sección $A$.

\section{Viga sometida a su propio peso}

\section{Canto variable}

Una viga de canto variable (sin alma) sometida a su propio peso no es más que la conjunción de un arco y un tirante. Si suponemos ambos realizados con el mismo material, la solución obtenida para el arco vale para la viga sin más que considerar el tirante como el mismo arco invertido. Las componentes horizontales se equilibran entre sí en los apoyos, resultando como reacción la suma de las componentes verticales. La solución que hemos obtenido más arriba permite obtener la máxima viga según la esbeltez utilizada, como queda descrito a continuación.

Denominamos esbeltez $\Gamma$ al cociente entre luz y canto, que, considerando [6], [7], y según se ve en la figura, vale para los valores máximo de $x, y, \theta$

$$
\begin{aligned}
& \Gamma=x / y \\
& \Gamma=\theta / \ln (1 / \cos \theta)
\end{aligned}
$$

Antes de continuar definimos un conjunto fundamental de términos (tal como se expresan en el texto principal de este artículo):

Al valor $\sigma / \varrho$, que, como se ve es de importancia primordial, le asignamos el nombre: Alcance del Material (es una longitud, y responde a la máxima longitud que podría alcanzar una barra recta de sección constante del

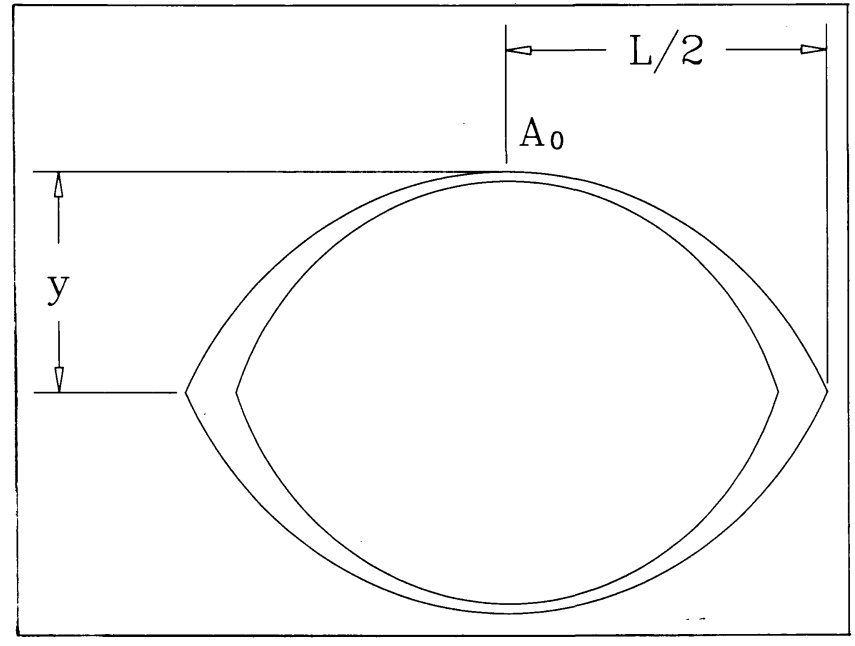

Fig. 5.-Viga de canto variable. material puesta en vertical, apoyada en uno de sus extremos, y sometida a su propio peso, ignorando problemas de estabilidad, etc.). (Para su uso en fórmulas sucesivas recuérdese la expresión [3]).

Asimismo definimos:

C que será la máxima luz lograda con la solución estructural, y supone la adopción de un esquema estructural, de una esbeltez de la solución, y de un material. A dicha magnitud (es una longitud) la denominamos Alcance de la solución estructural.

w para el valor $2 \theta=2 x / R_{0}=1 / R_{0}$

$\Omega$ para el producto entre w y esbeltez, valor al que denominaremos factor de alcance del esquema estructural, que si bien es función de la esbeltez, depende relativamente poco de ésta, como veremos más adelante, siendo básicamente un factor de forma dependiente de la tipología elegida (del esquema)

$$
\Omega=w \Gamma
$$


Resulta, considerando [6]:

$$
\begin{aligned}
\theta & =1 / 2 R_{0} \\
C & =2 R_{0} \theta=w R_{0}=\Omega / \Gamma R_{0}
\end{aligned}
$$

Es decir, el Alcance de la solución estructural (la máxima luz alcanzable) es igual al Alcance del material que se emplea en ella, multiplicado por el factor de alcance del esquema, y dividido por la esbeltez de la forma utilizada.

En el texto principal de este artículo incluimos una tabla de alcances de soluciones que usan el esquema "Viga estricta de canto variable", según su esbeltez, así como de los correspondientes factores de alcance.

\section{Canto constante}

Si consideramos una viga de canto constante y sección variable, el planteamiento puede ser como sigue:

Sean la Ley de Momentos en la viga M, la Ley de cortantes $\mathrm{T}$, la Ley de cargas q.

Considerense positivos los signos de las leyes de la figura. Por equilibrio es sabido que:

$$
q=d T / d x
$$

e igualmente

$$
q=-d^{2} M / d x^{2}
$$

es decir,

$$
\begin{aligned}
& \mathrm{q}=\mathrm{T}^{\prime} \\
& \mathrm{T}=-\mathrm{M}^{\prime}
\end{aligned}
$$

Por otro lado, al tratarse de una viga sometida a peso propio puede conocerse q como resultado de las secciones utilizadas. Si consideramos el origen de coordenadas en el centro del vano, de cortante nulo y sección igual a dos áreas $A_{0} / 2$ cada una, a distancia $z$, más alma (o barras a $45^{\circ}$ ) para el cortante, resultará en toda otra sección el valor, en cordones:

$$
A / A_{0}=M / M_{0}
$$

debiendo añadirse la sección de alma precisa por cortante.

Para resistir un momento $M$ es precisa un área total en cordones (o un volumen de material por unidad de longitud):

$$
A_{M}=2 M z \sigma
$$

siendo $z$ el brazo entre cordones (distancia entre centros de gravedad de las áreas), que suponemos constante. Aunque no es estrictamente igual al canto total de la pieza, es de un valor próximo al mismo. En lo sucesivo usaremos para el término canto el valor de $z$.

Es algo menos evidente el área precisa para resistir un cortante $T$ sin precisar más sobre el tipo de alma. Sin embargo, tanto si suponemos alma triangulada con barras a $45^{\circ}$, como si suponemos alma continua (considerando el criterio de rotura de Tresca, y asumiendo que la sección completa tiene un área muy relevante en alas respecto del área en alma, como en secciones en doble $\mathrm{T}$ ), el dimensionado estricto del alma requiere un volumen de material que, por unidad de longitud vale:

$$
A_{T}=2 T / \sigma
$$

de este modo la carga actuante vale

$$
\begin{aligned}
& q=q_{T}+q_{M}=\varrho\left(A_{T}+A_{M}\right) \\
& q=2 \varrho / \sigma(M / z+T)
\end{aligned}
$$


de donde resulta la ecuación diferencial de segundo orden

$$
M / z-M^{\prime}+M^{\prime \prime} \sigma / 2 \varrho=0
$$

Podemos resolverla suponiendo una solución singular exponencial de la forma

$$
M=k e^{c x}=k \exp (c x)
$$

con constantes $\mathrm{k}$ y c. La anterior ecuación se transforma en:

$$
k\left(1 / z-c+c^{2} \sigma / 2 \varrho\right) \exp (c x)=0
$$

que se verifica para raices de

$$
c^{2}-2 \varrho / \sigma c+2 \varrho / \sigma z=0
$$

Tales raíces son:

$$
\begin{aligned}
& c=\frac{\varrho}{\sigma} \pm \sqrt{\left.\left(\frac{\varrho}{\sigma}\right)^{2}-\frac{2 \varrho}{\sigma z}\right)}= \\
& =\varrho / \sigma(1 \pm \sqrt{1-2 \sigma / z \varrho})
\end{aligned}
$$

Como $z / 2<\sigma / \varrho$ el determinante es siempre negativo, de modo que escribimos

$$
c=\varrho / \sigma(1 \pm \mathrm{i} \sqrt{(2 \sigma / \varrho z-1)}
$$

y la solución general será de la forma

$$
M=k_{1} e^{c 1 x}+k_{2} e^{c 2 x}
$$

siendo $c 1$ y $c 2$ raices imaginarias conjugadas por lo que

$$
\begin{aligned}
& M=k_{1} e^{(\alpha+\beta) j x}+k_{2} e^{(\alpha-i \beta) x} \\
& M=e^{\alpha x}+\left(k_{1} e^{i \beta x}+k_{2} e^{-i \beta x}\right) \\
& M=e^{\alpha x}\left[\left(k_{1}+k_{2}\right) \cos \beta x+i\left(k_{1}-k_{2}\right) \operatorname{sen} \beta x\right]
\end{aligned}
$$

y tomando como valores de las constantes valores conjugados en el campo complejo, obtenemos la traza de la solución en el campo real, que es la que nos interesa:

$$
M=e^{x_{e} / \sigma}\left[A \cos \left(x \varrho / \sigma \sqrt{\left(2 \sigma / z \varrho^{-1}\right)}\right)+B \operatorname{sen}\left(x \varrho / \sigma \sqrt{\left(2 \sigma / Z \varrho^{-1}\right)}\right)\right]
$$

Por sencillez de notación utilizaremos

$$
\begin{aligned}
\mathrm{R} & =d \varrho, \mathrm{y} \\
\theta & =1 / \sqrt{(2 \mathrm{R} / \mathrm{z}-1)}=1 / \sqrt{(2 \Gamma / \mathrm{w}-1)}
\end{aligned}
$$

usando

$$
\begin{aligned}
& z=1 / \Gamma=w R / \Gamma \\
& v=x / R
\end{aligned}
$$

y trabajaremos con la expresión adimensional de la forma, $v$, que varía entre 0 en el centro de la viga a w/2 en el apoyo. De este modo, y como

$$
\begin{aligned}
\mathrm{df} / \mathrm{dx} & =\mathrm{df} / \mathrm{dv} d \mathrm{v} / \mathrm{dx}=1 / \mathrm{R} \mathrm{df} / \mathrm{dv} \\
\mathrm{M} & =\mathrm{e}^{v}(A \cos v / \theta+B \operatorname{sen} v / \theta) \\
T & =-1 / R \mathrm{e}^{v}((A+B / \theta) \cos v / \theta+(B-A / \theta) \operatorname{sen} v / \theta)
\end{aligned}
$$


Si consideramos el origen de ejes en el punto de máximo momento positivo (en centro de vano si hay simetría), donde el cortante es nulo,

$$
\begin{aligned}
& T_{0}=0=-(A+B / \theta) / R \\
& B=-A \theta=K
\end{aligned}
$$

usamos $\mathrm{K}$ como constante a partir de aquí.

$$
\begin{aligned}
\mathrm{M} & =\mathrm{K} \mathrm{e}^{\mathrm{v}}(-1 / \theta \cos \mathrm{v} / \theta+\operatorname{sen} \mathrm{v} / \theta) \\
\mathrm{T} & =-\mathrm{K} / \mathrm{Re}^{\mathrm{v}}\left(\left(1 / \theta^{2}+1\right) \operatorname{sen} \mathrm{v} / \theta\right) \\
\mathrm{q} & =-\mathrm{K}^{2} \mathrm{R}^{2} \mathrm{e}^{\mathrm{v}}\left(\left(1+1 / \theta^{2}\right) \operatorname{sen} \mathrm{v} / \theta+\left(1 / \theta+1 / \theta^{3}\right) \cos \mathrm{v} / \theta\right)
\end{aligned}
$$

es decir,

$$
\begin{aligned}
M & =K e^{v}(\operatorname{sen} v / \theta-1 / \theta \cos v / \theta) \\
T & =-K / R\left(1+1 / \theta^{2}\right) e^{v} \operatorname{sen} v / \theta \\
q & =-K / R^{2}\left(1+1 / \theta^{2}\right) e^{v}(\operatorname{sen} v / \theta+1 / \theta \cos v / \theta)
\end{aligned}
$$

Veamos el valor de la constante $K$ de integración: en el punto origen

$$
q=q_{0}=-K / R^{2}\left(1+1 / \theta^{2}\right) 1 / \theta=A_{o e}
$$

siendo $A_{0}$ el área total en tal punto, por lo que

$$
K=-A_{\circ} \varrho R^{2} \theta /\left(1+1 / \theta^{2}\right)
$$

y considerando que

$$
\left(1+1 / \theta^{2}\right)=2 \mathrm{R} / \mathrm{z}
$$

resulta, en definitiva,

$$
\begin{aligned}
q & =A_{o} \varrho \theta e^{v}(\operatorname{sen} v / \theta+1 / \theta \cos v / \theta) \\
T & =A_{o} \varrho \theta R e^{v} \operatorname{sen} v / \theta \\
M & =A_{0} \varrho \theta R z / 2 e^{v}(\operatorname{sen} v / \theta-1 / \theta \cos v / \theta)[12]
\end{aligned}
$$

Ahora bien, podemos utilizar, como anteriormente, $I=$ wR, siendo $\Gamma=I / z$ y $L=C$ la luz máxima alcanzada por la solución, de modo que tendríamos para la expresión del Momen. to, en función del valor del canto:

$$
\begin{aligned}
z & =1 / \Gamma=w R / \Gamma \\
M & =A_{0} \varrho \theta w R^{2} / 2 \Gamma e^{v}(\operatorname{sen} v / \theta-1 / \theta \cos v / \theta)
\end{aligned}
$$

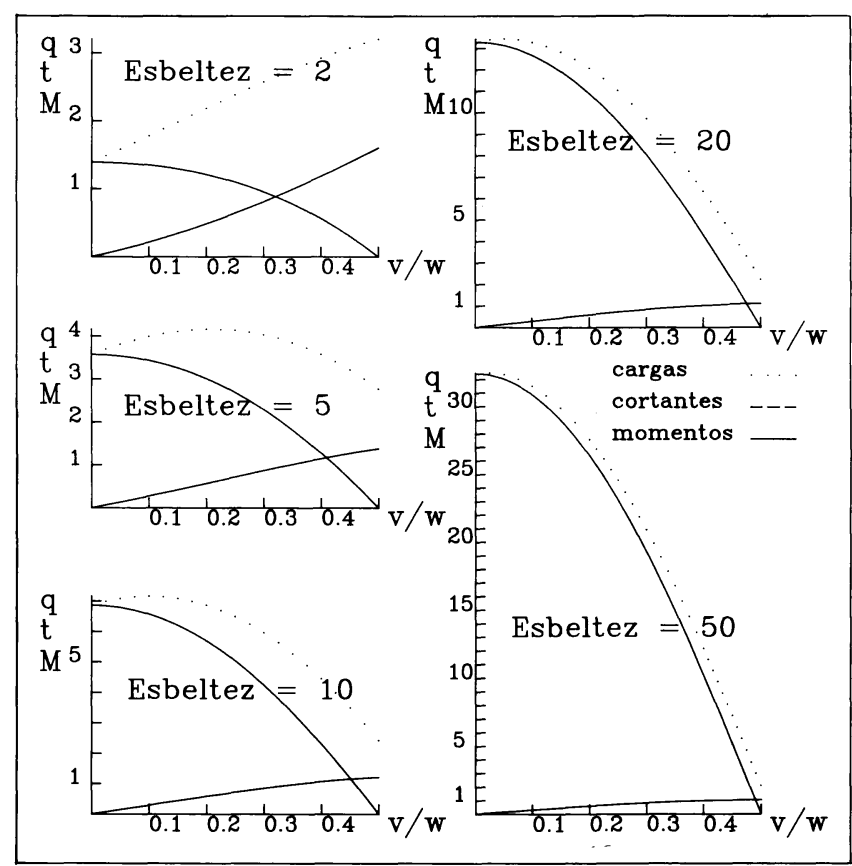

Fig. 7.-Diagramas de $q, T, M$ según esbeltez (factores de escala según texto).

[13]

Si queremos determinar ahora el alcance de la estructura (su luz máxima), por ejemplo para el caso de estar simplemente apoyada en sus dos extremos, nos bastará establecer que el momento es nulo en el apoyo, en la coordenada correspondiente a la luz mitad, es decir,

$$
M(v)=0
$$

para

$$
v=(L / 2) / R=w R / 2 R=w / 2
$$

de modo que la expresión [13] permite resolver $w$ para cada valor de $\Gamma$.

Resolviendo tal expresión para los valores usados en el caso de la viga de sección variable (arco-tirante) podemos establecer una segunda tabla, que figura análogamente en el texto principal, correspondiente a los alcances de la viga de canto constante. 
En la figura 7 se incluyen las gráficas de $q / A_{\circ} \varrho \theta, T / A_{\diamond} \varrho \theta R$ y $M / A_{\diamond} \varrho \theta R z / 2$ para diversas esbelteces. Cabe señalar finalmente que la solución de viga de canto constante es más eficiente que la de sólo cordones con canto variable a partir de una esbeltez en torno al valor de 7 . Si se evalúa el valor de coincidencia con los medios del análisis anterior, la esbeltez que separa los campos de validez de las dos tipologías (supuesto el uso del mismo material en ambas) es

$$
\Gamma_{\mathrm{t}}=7,4273
$$

siendo los cordones paralelos más apropiados para esbelteces mayores que $\Gamma_{\mathrm{t}}$

\section{publicaciones del ICCET/CSIC}

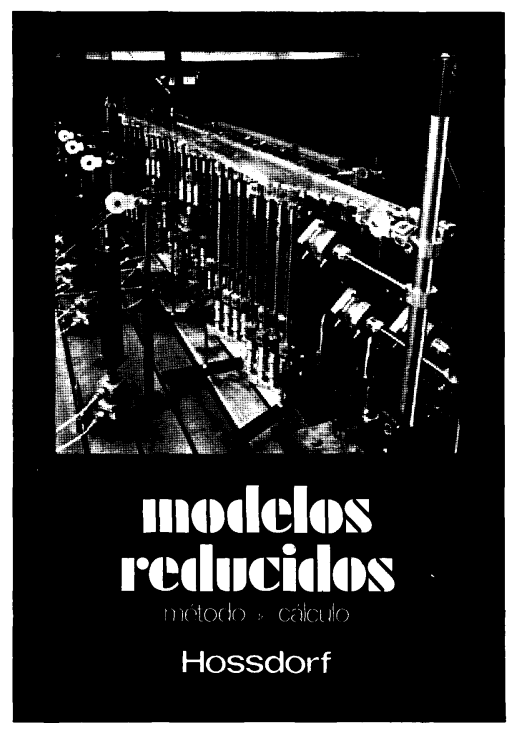

Modelos reducidos. Método de cálculo

H. Hossdorf, Ingeniero Civil

La técnica de los ensayos en modelos reducidos de estructuras sufre hoy dia una decisiva metamorfosis. Hasta hace poco era un medio más bien de artesania, que no siempre era tomado en serio por los académicos teorizantes para comprender el comportamiento resistente de las estructuras complejas $y$ al que se acudió las más de las veces, como a un último remedio debido a sus indiscutibles insuficiencias. Sin embargo, en poco tiempo y gracias a su conexión con los ordenadores digitales, se ha transformado en un instrumento cientificamente valioso, diaria del Ingeniero Proyectista.

Un volumen encuadernado en cartoné plastificado con lomo de tela de $17 \times 24 \mathrm{~cm}$, compuesto de 250 páginas, 158 figuras $y$ fotografias.

Precios: 1.800 ptas.; \$ USA 26.00.

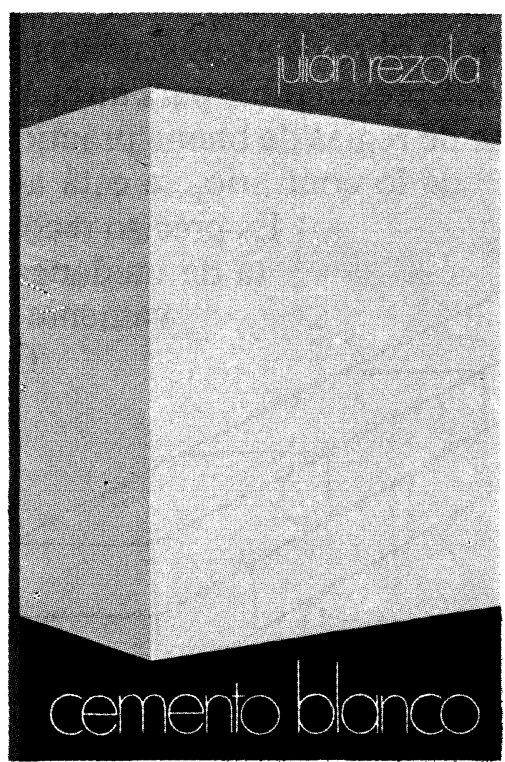

\section{Cemento blanco}

Julián Rezola

Ingeniero Químico Dipl. I. Q. S.

Sabido es que existe una extensa y documentada bibliografia sobre el cemento gris: en cambio, no
puede decirse lo mismo acerca del cemento portland blanco, ya que los escritos existentes se refieren tan sólo a algunas peculiaridades que le distinguen de aquél.

El autor nos ofrece sus profundos conocimientos y su larga experiencia tanto en laboratorio como en fabricación.

La parte descriptiva del libro se complementa con gráficos, diagramas y fotografias de gran utilidad, destinados a conseguir la aplicación apropiada de este aglomerante.

Un volumen encuadernado en cartoné policerado, de $17,4 \times 24,3 \mathrm{~cm}$, compuesto de 395 páginas, numerosas figuras, tablas $y$ abacos.

Precios: España, 1.700 ptas.; extranjero, \$ 24.

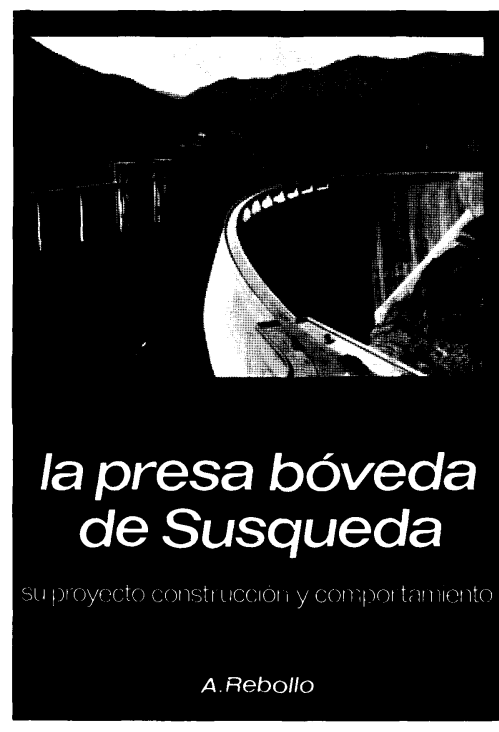

La presa bóveda de Susqueda

A. Rebollo,

Dr. Ingeniero de Caminos

El esfuerzo del constructor de presas se sitúa por su pretensión de perennidad, a contracorriente de las tendencias de la civilización actual, caracterizada por to fungible. Pueden evocarse las 10.000 grandes. presas en funcionamiento o en construcción que están envejeciendo y reclaman los cuidados gerontologicos para mantener y perfeccionar su servicio y garantizar su inalienable pretension de perennidad. En la medida en que todas nuevas obras, grandes o pequeñas, son portadoras de riesgos ecológicos $y$, a veces, catastroficos, que aumentan con el envejecimiento, la gerontologia de las presas es todo un emplazo. La acción adelantada de Arturo Rebollo en este terreno marca un camino a seguir para todos los que aman su propia obra con Un volumencu con lomo de con lomo de tela, de $18 \times 24,5 \mathrm{~cm}$, compuesto de 408 páginas, 330 figuras y fotografias y 39 tablas. Precios: 1.700 ptas.; extranjero, \$ USA 24.00 